\title{
Custo total de propriedade (TCO): Estudo de caso em uma instituição de ensino superior privada do Rio Grande do Sul
}

\begin{abstract}
Alvaro Luiz Neuenfeldt Júnior
Mestrado em Engenharia de Produção pela Universidade Federal de Santa Maria -

UFSM

Representante discente no colegiado do PPGEP da Universidade Federal de Santa

Maria - UFSM

Avenida Roraima, 1000. Camobi. Santa Maria/RS. CEP 97105-900

E-mail: alvjr2002@hotmail.com

Charlene Coser Dalcol

Mestrado em Engenharia de Produção pela Universidade Federal de Santa Maria -

UFSM

Coordenadora da Assessoria de Relações Públicas da Faculdade Palotina de Santa Maria - FAPAS

Avenida Presidente Vargas, 115. Patronato. Duque de Caxias. Santa Maria/RS. CEP:

97020-001

E-mail: charlenedalcol@gmail.com
\end{abstract}

Andreas Dittmar Weise

Doutorado em Engenharia Civil pela Universidade Federal de Santa Catarina - UFSC Professor Adjunto da Universidade Federal de Santa Maria - UFSM Avenida Roraima, 1000. Camobi. Santa Maria/RS. CEP: 97105-900

E-mail: andreas.weise@ufsm.br

Julio Cezar Mairesse Siluk

Doutorado em Engenharia de Produção pela Universidade Federal de Santa Maria -

UFSM

Coordenador do PPGEP da Universidade Federal de Santa Maria - UFSM Avenida Roraima, 1000. Camobi. Santa Maria/RS. CEP: 97105-900

E-mail: jsiluk@ufsm.br

\section{RESUMO}

A inserção de técnicas para o gerenciamento financeiro empresarial, como o Custo Total de Propriedade (TCO), servem como esclarecedores quanto a verificação do papel dos fatores qualitativos presentes na negociação com os fornecedores, possibilitando assim a redução dos recursos empregados para determinado fim. Nesse contexto, nesse artigo objetiva-se mostrar a aplicação do Custo Total de Propriedade na reestruturação do setor de compras de TI de uma Instituição de Ensino Superior privada localizada no Rio Grande do Sul, partindo inicialmente da expansão de um de seus laboratórios de informática e da criação de uma nova sala para a modalidade de Ensino à Distância, examinando-se a forma como as solicitações de serviços e compra 
Alvaro Luiz Neuenfeldt Júnior, Charlene Coser Dalcol, Andreas Dittmar Weise, Julio Cezar Mairesse Siluk

de materiais são realizados atualmente. $O$ estudo revela que foi possível perceber que pode se obter ganhos de, aproximadamente, 38\% em relação ao valor inicial proposto pela instituição, além da padronização do atual processo de compras para esse tipo de atividade.

Palavras-Chave: Custo Total de Propriedade. Gestão Financeira. Instituições de Ensino Superior.

\section{Total cost of ownership (TCO): A case study a private superior teaching institution of Rio Grande do Sul}

\section{ABSTRACT}

The insertion of business financial management techniques, such as the Total Cost of Ownership (TCO), serve as enlightening as the role of qualitative factors present in negotiating with suppliers, thus enabling it to be feasible to reduce the resources employed for particular purpose. Thus, this article aims to show the application Total Cost of Ownership to restructure the industry purchases for a private higher education institution located in Rio Grande do Sul, starting initially case of the expansion of one computer labs and the creation of a new room for distance learning mode, through the study of the current way that service requests and purchase of materials are carried out. Finally, it was possible to realize gains can be obtained approximately $38 \%$ in relation to the initial value proposed by the institution, besides the standardization of the current process of shopping for this kind of activity.

Keywords: Total Cost of Ownership. Financial Management. Higher Education Institution.

\section{INTRODUÇÃO}

Inseridas em um mercado que ao longo dos anos vem se tornando cada vez mais competitivo, as Instituições de Ensino Superior (IES) privadas brasileiras demandam ferramentas e técnicas que compreeendem a formulação de estratégias coerentes com os fatos e dados encontrados em relação a realidade do contexto setorial e empresarial, ainda mais quando se trata das questões inerentes a gestão dos 
Alvaro Luiz Neuenfeldt Júnior, Charlene Coser Dalcol, Andreas Dittmar Weise, Julio Cezar Mairesse Siluk

recursos financeiros, buscando focar a atenção nos verdadeiros fatores críticos que influenciam o seu desempenho (SANTOS et al., 2010; LIMA FILHO et al., 2011).

Estudos a respeito dessa temática ganharam ainda mais força devido ao aumento da parcela dos custos de bens e serviços adquiridos de maneira externa às organizações, resultando na necessidade de maior eficácia dos controles de entrada, pois estes podem ser considerados como um dos elementos decisivos para a determinação do sucesso competitivo de uma organização (OGDEN; ZSIDISIN, 2003).

Ao longo dos anos ferramentas para a gestão dessas atividades foram sendo desennvolvidas, a fim de mitigar os possíveis efeitos adversos inerentes aos tradicionais formatos paradigmáticos de pensamento (DEGRAEVE et al., 2005; FEIMAN 2009). Dentre estas, se encontra em posição de destaque o Custo Total de Propriedade (TCO), por tratar a temática dos custos sob a ótica da gestão do ciclo de vida de um produto, projeto ou serviço da empresa, a fim de se entender o papel dos diversos elementos qualitativos das atividades envolvidas na negociação com os fornecedores, visto a possibilidade de otimizaros custos investidos com a solicitação de serviços de assistência técnica e possíveis falhas dos produtos que exigam a sua manutenção antes do tempo previsto (ZACHARIASSEN; ARLBJØRN, 2011).

A partir desses aspectos evolutivos, o estudo do TCO pode ser vinculado ao aumento de controle dos custos relativos à Tecnologia da Informação (TI) nas organizações, independente da sua natureza jurídica, de forma a ampliar os horizontes dos conhecimentos aplicados às atividades de gestão desse tipo de ativo (FEIMAN, 2009). Especificamente em relação às IES, a sua utilização pode ser justificada pela falta de controle existente em relação às atividades de compras para materiais, o que gera dispêndios financeiros extras, além de enfraquecer o nível de relacionamento existente com os fornecedores dos produtos e serviços, visto o papel proeminente na gestão empresarial do controle financeiro acerca dos dispêndios realizados com a aquisição de bens ou contratação de serviços, consideradas por Cousins et al. (2008) como formadoras de, no mínimo, $50 \%$ dos custos da empresa. 
Alvaro Luiz Neuenfeldt Júnior, Charlene Coser Dalcol, Andreas Dittmar Weise, Julio Cezar Mairesse Siluk

Ao propor esse tipo de aplicação, por menores que sejam as reduções obtidas nos custos, há uma possibilidade de se conseguir impactar de maneira significativa os resultados operacionais da empresa em questão, sendo, portanto, uma área de alto potencial de interesse tanto no âmbito de estudos acadêmicos-científicos quanto do ambiente profissional. Assim, o presente artigo objetiva mostrar a aplicaçãodo Custo Total de Propriedade para reestruturar o setor de compras de TI de uma Instituição de Ensino Superior privada localizada no Rio Grande do Sul, partindo inicialmente do caso da expansão de um de seus laboratórios de informática e da criação de uma nova sala para a modalidade de Ensino à Distância (EAD).

\subsection{Custo Total de Propriedade}

Um dos paradigmas mais destacados em relação ao sistema de custos de uma empresa é a orientação dos setores de compras quanto ao seu posicionamento estratégico, focado simplesmente em produzir ganhos financeiros através da redução dos preços dos bens e serviços contratados, em detrimento a outros fatores considerados como preponderantes no momento da contratação, de maneira a não explorar todo o potencial estratégico dessa atividade em relação à própria empresa e aos fornecedores (TOMKINS, 2001; KULMUALA, 2004).

Visto o seu objetivo de otimizar os recursos utilizados para o pleno funcionamento do sistema de gestão dos custos organizacionais, o TCO foi definido para abordar o contexto das atividades envolvidas com a aquisição de bens, partindo de um elevado grau de abstração até o seu detalhamento, levando em conta o planejamento voltado para a usuabilidade das tecnologias até o seu respectivo papel no momento de ser implementado (DEGRAEVE et al., 2005; FEIMAN, 2009).

A partir de estudos realizados com gerentes de diversos ramos de atuação empresarial, Ellram (1993) e, posteriormente, Degraeve et al. (2005) e Feiman (2009) revelam que a abordagem do TCO pode ser classificada de três maneiras: quanto à formalização, ao nível de padronização e as características das informações. Para a 
Alvaro Luiz Neuenfeldt Júnior, Charlene Coser Dalcol, Andreas Dittmar Weise, Julio Cezar Mairesse Siluk

formalização, a aplicação do modelo pode ser observada sob duas óticas distintas em que, na primeira, definida como informal, os fatores de custos são tratados por meio do conhecimento empírico a respeito da forma com que interferem no sistema, desconhecendo o real impacto dessas ações no custo total. Já a segunda abordagem leva em consideração o caráter formal e explícito no qual as informações estão relacionadas através de métodos e diretrizes escritas e documentadas por intermédio de procedimentos realizados para as diversas atividades existentes no momento da efetivação da solicitação de um produto (WOUTERS et al., 2005; PARK et al., 2010).

O TCO aplicado em uma empresa pode ser classificado também quanto à forma com que ocorre o nível de padronização, podendo ser modelos basicamente normalizados, onde a classificação dos grupos de fatores existentes acontece de maneira comum através da repetida utilização dos mesmos procedimentos para diferentes tipos de compras, necessitando de poucas modificações nas métricas adotadas, se caracterizando pela fácil manipulação por parte dos usuários e rapidez com que retorna resultados à empresa.

Em contrapartida, existem também os modelos customizados, focados na construção de sistemas de compras específicos para determinadas situações relacionadas ao usuário da ferramenta, demandando para isso tempo, recursos e conhecimento acerca dos problemas a serem analisados (HURKENS et al. 2006). Porém, existem pesquisas realizadas por Ellram e Zsidisin (2002) que indicam uma plena satisfação das empresas que investiram na customização pois, apesar das demandas iniciais, as funcionalidades específicas obtidas compensam a médio e longo prazo os usuários.

A terceira forma de classificação da aplicação do TCO em uma empresa é quanto às características das informações utilizadas, podendo ser divididas em três aspectos. O primeiro está baseado no custeio direto em relação a cada compra específica, ocorrendo à inserção das informações de maneira real e direta caso a caso. A segunda está relacionada com a alocação dos custos, por meio de uma ferramenta 
Alvaro Luiz Neuenfeldt Júnior, Charlene Coser Dalcol, Andreas Dittmar Weise, Julio Cezar Mairesse Siluk

capaz de classificar quais são mais relevantes para o sistema, como é o caso encontrado para o custeio por atividade $(A B C)$, onde os itens não selecionados para inspeção ficam isentos da adição do TCO para a tarefa determinada. Por fim, o último modelo é tratado em conjunto com outros aspectos qualitativos, através do cálculo denominado por fator de custo total, geralmente relacionado ao nível de desempenho percebido por parte do comprador em relação ao fornecedor, através da série histórica de acontecimentos relativos aos quesitos avaliados (GARFAMY, 2006).

Potencialmente, independente do formato de TCO adotado para a gestão dos recursos da empresa, este retorna aos envolvidos contribuições significativas no que tange a mudanças na maneira com que as atividades de solicitação de bens e serviços são realizadas, por meio, principalmente, da mudança de cultura das pessoas e dos procedimentos adotados.

\section{INSTITUIÇÕES DE ENSINO SUPERIOR}

Por se tratarem de organizações que visam a prestação de serviços focados nas atividades de ensino, pesquisa e extensão, as IES tem por dever contribuir na formação profissional de jovens e adultos, a partir de um sistema complexo, diversificado e em constante expansão, mas que a gestão deve funcionar da mesma maneria que a encontrada em empresas comerciais ou industriais (HANSEN, 2001). Dias Sobrinho (2003) define que o modelo de gestão adotado pelas IES pode se diferenciar conforme as suas características e o ambiente em que atuam.

Independente da estrutura adotada, as IES devem inserir em sua estratégia organizacional a preocupação com o desenvolvimento de atividades no âmbito social, suprindo em certas situações as deficiências de atendimento do poder público em algumas localidades (BOK, 2003; KRAEMER, 2003). Devido a esse e outros fatos, 0 formato de gestão exige uma dupla dedicação quanto ao modelo estratégico adotado pela administração, abordando tanto questões relativas ao ensino quanto ao 
Alvaro Luiz Neuenfeldt Júnior, Charlene Coser Dalcol, Andreas Dittmar Weise, Julio Cezar Mairesse Siluk

atendimento das demandas relativas a estrutura física e de recursos humanos, cabendo a IES encontrar meios de atender essas demandas visando o seu pleno desenvolvimento (MCCHLERY; ROLFE, 2004).

Em relação à administração dos recursos de compras, Merlo e Beuren (2004) citam que a gestão econômica e financeira de uma instituição de ensino é considerada como atividade-meio, por estabelecer um suporte às atividades que realmente agregam e representam os objetivos da organização, mas que, independente dessa classificação, são elencadas como de alta necessidade e importância, principalmente quando vinculadas a compra de materiais e a solicitação de serviços.

Progressos vêm sendo notados nas formas com que os custeios são realizados em IES, adotando-se técnicas capazes de clarificar a forma com que a alocação dos recursos está para a projeção dos custos de aquisição, a fim de auxiliar diretamente na proposição de estudo relativos a criação e encerramento de cursos, precificação dos produtos aos clientes e de acompanhamento do nível em que se encontra a saúde financeira da empresa.

\subsection{Competitividade}

O cenário atual das organizações tende para que estas se envolvam cada vez mais em ambientes altamente competitivos, seja em relação aos seus concorrentes considerados como diretos como, até mesmo, para os indiretos (PORTER, 2009; HITT; IRELAND; HOSKISSON, 2012). Dessa forma, existe a necessidade de criarem valor nas atividades desenvolvidas para a concepção de um produto ou serviço, tanto no âmbito externo a organização quanto setorial e interno, atendendo assim as expectativas dos seus consumidores, de maneira a atrair, por consequência, novos investimentos para a sua capacitação e aprimoramento, gerando novos valores e repassando estes ao mercado (LOBATO, 2006; ZOGBI, 2008).

Pode-se dizer, conforme pressupostos embasados em Porter (2009), que a competitividade setorial vai muito além da análise dos tradicionais adversários, e sim 
Alvaro Luiz Neuenfeldt Júnior, Charlene Coser Dalcol, Andreas Dittmar Weise, Julio Cezar Mairesse Siluk

depende de uma série de outras questões chamadas de formas competitivas, que tangem desde os clientes até fornecedores, potenciais entrantes e possíveis produtos substitutos, sendo agentes no comportamento dos vetores da lucratividade da empresa e do posicionamento estratégico da empresa (DI SERIO; VASCONCELLOS, 2009).

\section{METODOLOGIA}

A pesquisa tem por base de investigação o método quantitativo, para melhor elucidação do problema ao qual se está abordando a fim de decodificar e descrever em números os significados dos dados coletados no sistema estudado (LAKATOS; MARCONI, 2001; MINAYO, 2010). A necessidade de compreender algum fator considerado de alta complexidade faz com que hajam pesquisas relativas ao tema, de forma a se investigar as possíveis causas e características significativas dos eventos relacionados, buscando soluções compatíveis com o ambiente ao qual se está inserido (YIN, 2009). Dessa forma, o estudo de caso tem por lógica esclarecer uma ou mais decisões a serem tomadas, analisando o motivo pelo qual estas estão sendo estudadas, a partir da formulação, com alguma precisão, das questões mais significantes a respeito do problema (GIL, 2010).

Como referência para o estudo escolheu-se uma IES privada instalada no Estado do Rio Grande do Sul, devido à facilidade de acesso às informações necessárias e a disponibilidade demandada pelos gestores para que tal pesquisa fosse realizada. Como base para certificação a respeito das técnicas e ferramentas utilizadas no estudo, foi proposta inicialmente a fundamentação teórica sobre Custo Total de Propriedade, devido a necessidade de maior entendimento sobre a sua funcionalidade e grau de adequação a problemática proposta, facilitando a compreensão das possibilidades de melhoria do sistema de compras para equipamentos e serviços relacionados à área de TI da IES escolhida, além de ser um método flexível para esse tipo de aplicação prática, 
Alvaro Luiz Neuenfeldt Júnior, Charlene Coser Dalcol, Andreas Dittmar Weise, Julio Cezar Mairesse Siluk

visando ganhos não só no âmbito financeiro, e sim de maneira gerencial, conforme a situação em que se encontra o sistema atual.

Para a coleta dos dados foi aplicado um disgnóstico semi-estruturado, subdividido em três eixos temáticos (processo atual de compra, equipamentos atuais e prospecções futuras) e submetido por meio de entrevistas aos funcionários e gestores de Tecnologia da Informação ( $\mathrm{TI})$ e corpo diretivo da empresa. A análise dos dados foi realizada pelos métodos formais e customizados, juntamente com o custeio direto, devido à adequação ao problema proposto, pois a série histórica de compras da IES para os produtos de $\mathrm{Tl}$ é relativamente pouco estruturada.

Ao final, de modo a contemplar o objetivo da pesquisa e expressar os resultados de forma mais evidente, o TCO foi separado em duas etapas distintas: a primeira contempla questões relativas aos dispêndios financeiros para as solicitações dos equipamentos ou serviços, chamada de TCO custo; e a segunda está de acordo com a padronização do atual fluxo de informações das mesmas, a partir do parecer dos colaboradores envolvidos com as áreas de compras e TI da empresa, denominado de TCO processos.

\section{APLICAÇÃO E RESULTADOS}

Inicialmente foi disponibilizado pela IES o orçamento máximo de $\mathrm{R} \$ 100.000,00$ destinado exclusivamente a compra de equipamentos de informática (TCOi=100000). Posteriormente houve o levantamento das características dos dois laboratórios, definidas no estudo por SALAS, sendo o primeiro (SALA 1) relativo a expansão do laboratório de pesquisas técnico-científicas para os discentes, dobrando a sua capacidade para 40 lugares, e outro (SALA 2) capaz de atender a demanda por aulas sob o formato de Ensino à Distância (EAD), exigindo o atendimento simultâneo de 30 usuários. 
Alvaro Luiz Neuenfeldt Júnior, Charlene Coser Dalcol, Andreas Dittmar Weise, Julio Cezar Mairesse Siluk

Buscando-se um maior entendimento acerca do sistema e o status ao qual está inserido no contexto da IES, inicialmente houve o levantamento dos atuais equipamentos existentes, por meio do diagnóstico citado na seção sobre a metodologia, de acordo com as suas características de usuabilidade, a fim de estimar os seus preços de mercado nos dias atuais, conforme mostra a Tabela 1.

Tabela 1 - Equipamentos atualmente existentes na SALA 1

\begin{tabular}{|c|c|c|c|c|}
\hline \multicolumn{5}{|c|}{ SALA 1} \\
\hline Descrição & Status & Qde. & $\begin{array}{l}\text { Preço } \\
\text { Un. (R\$) }\end{array}$ & $\begin{array}{c}\text { Preço } \\
\text { Total (R\$) }\end{array}$ \\
\hline \multicolumn{5}{|c|}{ Desktop } \\
\hline $\begin{array}{c}\text { Processador Intel Core } 2 \\
\text { Duo 2.4 GHz; } 3 \text { GB; HD } \\
160 \mathrm{~GB} \\
\end{array}$ & Usado & 20 & 600,00 & $12.000,00$ \\
\hline \multicolumn{5}{|c|}{ Monitor } \\
\hline LCD 17" & Usado & 20 & 200,00 & $4.000,00$ \\
\hline \multicolumn{5}{|c|}{ Servidor para aplicação de dados } \\
\hline $\begin{array}{c}\text { Processador Intel Core } 2 \\
\text { Quad Q6600; } 6 \text { GB; HD } \\
\text { 250 GB }\end{array}$ & Usado & 1 & 950,00 & 950,00 \\
\hline \multicolumn{5}{|c|}{ Switch } \\
\hline $\begin{array}{c}24 \text { portas } 10 / 100 \text { Mbps; } 3.2 \\
\text { GHz de banda }\end{array}$ & Usado & 1 & 150,00 & 150,00 \\
\hline \multicolumn{5}{|c|}{ Nobreak } \\
\hline 1300 Va com duas baterias & Usado & 4 & 250,00 & $1.000,00$ \\
\hline \multicolumn{5}{|c|}{$\begin{array}{ll}\text { Storage } \\
\end{array}$} \\
\hline Storage System & Usado & 1 & 800,00 & 800,00 \\
\hline \multicolumn{4}{|c|}{ Total } & $18.900,00$ \\
\hline
\end{tabular}

A SALA 1 possui equipamentos em plenas condições de uso que em valores atuais representam $R \$ 18.900,00$, mesmo que de alguma forma possam ser considerados como obsoletos para o mercado, enquanto que a SALA 2, conforme anteriormente mencionado, não possuía equipamentos a disposição.

De acordo com os pressupostos elaborados pela administração da IES em estudo, o indicador denominado por TCOd está definido para informar o total de 
investimentos necessários para a abertura de duas novas salas de informática, a partir do somatório total da compra de equipamentos novos, a partir da Equação (1),

$$
\operatorname{Tcod}=\sum_{x=1}^{n} \operatorname{Tcodx}\left\{\begin{array}{c}
x \in\{1,2\} \\
\operatorname{TCOd}>0 \\
\operatorname{TCOdx}=\sum_{\mathrm{i}=1}^{n} \sum_{\mathrm{e}=1}^{\mathrm{n}}(\mathrm{ITe})_{\mathbf{i}}\left\{\begin{array}{l}
\forall \mathrm{i} \in\{1,2, \ldots, \mathrm{n}\} \\
\forall \mathrm{e} \in\{1,2, \ldots, \mathrm{n}\}
\end{array}\right.
\end{array}\right.
$$

onde $x$ identifica cada um dos locais levados em consideração para o estudo, no caso TCOd1 é a SALA 1 e TCOd2 a SALA 2, relacionados diretamente com a soma dos $i$ itens levados em consideração como Desktops, Monitores, Servidores, Switch, Nobreak, Storage System e acessórios diversos, para os ITe equipamentos localizados em cada um destes.

A Tabela 2 mostra o total calculado TCOd de investimentos necessários para a abertura de duas novas salas de informática a partir da compra de todos equipamentos novos. 
Custo total de propriedade (TCO): Estudo de caso em uma instituição de ensino superior privada do Rio Grande do Sul

Alvaro Luiz Neuenfeldt Júnior, Charlene Coser Dalcol, Andreas Dittmar Weise, Julio Cezar Mairesse Siluk

Tabela 2 - Cálculo do TCOd relativo a compra de todos equipamentos novos

\begin{tabular}{|c|c|c|c|c|c|c|c|c|c|}
\hline \multicolumn{5}{|c|}{ SALA 1} & \multicolumn{5}{|c|}{ SALA 2} \\
\hline Descrição & Status & Qde. & $\begin{array}{c}\text { Preço } \\
\text { Un. (R\$) }\end{array}$ & $\begin{array}{l}\text { Preço } \\
\text { Total } \\
(\mathbf{R} \$)\end{array}$ & Descrição & Status & Qde. & $\begin{array}{c}\text { Preço } \\
\text { Un. (R\$) }\end{array}$ & $\begin{array}{l}\text { Preço } \\
\text { Total } \\
(\mathbf{R} \$)\end{array}$ \\
\hline \multicolumn{5}{|c|}{ Desktop } & \multicolumn{5}{|c|}{ Desktop } \\
\hline $\begin{array}{l}\text { Processador } \\
\text { segunda } \\
\text { geração } \\
\text { Intel Core } \\
\text { i3-2120 } 3.3 \\
\text { GHz, } 4 \\
\text { Threads; } 3 \\
\text { Mb; } 4 \text { GB; } \\
\text { HD 500GB }\end{array}$ & Novo & 40 & $1.550,00$ & $62.000,00$ & $\begin{array}{l}\text { Processador } \\
\text { segunda } \\
\text { geração } \\
\text { Intel Core } \\
\text { i3-2120 3.3 } \\
\text { GHz, } 4 \\
\text { Threads; } 3 \\
\text { Mb; } 4 \text { GB; } \\
\text { HD 500GB }\end{array}$ & Novo & 30 & $1.550,00$ & $46.500,00$ \\
\hline \multicolumn{5}{|c|}{ Monitor } & \multicolumn{5}{|c|}{ Monitor } \\
\hline LCD 18,5" & Novo & 40 & 300,00 & $12.000,00$ & LCD 18,5" & Novo & 30 & 300,00 & $9.000,00$ \\
\hline \multicolumn{5}{|c|}{ Servidor para aplicação de dados } & \multicolumn{5}{|c|}{ Servidor para aplicação de dados } \\
\hline $\begin{array}{c}\text { Processador } \\
\text { Intel Xeon } \\
\text { X3430 } 2.4 \\
\text { GHZ; } 6 \text { GB; } \\
\text { HD } 500 \text { GB }\end{array}$ & Novo & 1 & $2.900,00$ & $2.900,00$ & $\begin{array}{l}\text { Processador } \\
\text { Intel Xeon } \\
\text { X3430 } 2.4 \\
\text { GHZ; } 6 \text { GB; } \\
\text { HD } 500 \text { GB } \\
\end{array}$ & Novo & 1 & $2.900,00$ & $2.900,00$ \\
\hline \multicolumn{5}{|c|}{ Switch } & \multicolumn{5}{|c|}{ Switch } \\
\hline $\begin{array}{c}24 \text { portas } \\
10 / 100 \\
\text { Mbps; } 3.2 \\
\text { GHz de } \\
\text { banda }\end{array}$ & Novo & 1 & 150,00 & 150,00 & $\begin{array}{c}24 \text { portas } \\
10 / 100 \\
\text { Mbps; } 3.2 \\
\text { GHz de } \\
\text { banda }\end{array}$ & Novo & 2 & 250,00 & 500,00 \\
\hline $\begin{array}{c}24 \text { portas } \\
10 / 100 \\
\text { Mbps; } 3.2 \\
\text { GHz de } \\
\text { banda }\end{array}$ & Novo & 1 & 250,00 & 250,00 & & & & & \\
\hline \multicolumn{5}{|c|}{ Nobreak } & \multicolumn{5}{|c|}{ Nobreak } \\
\hline $\begin{array}{c}1300 \mathrm{Va} \\
\text { com duas } \\
\text { baterias }\end{array}$ & Novo & 8 & 500,00 & $4.000,00$ & $\begin{array}{c}1300 \mathrm{Va} \\
\text { com duas } \\
\text { baterias }\end{array}$ & Novo & 6 & 500,00 & $3.000,00$ \\
\hline \multicolumn{5}{|c|}{ Storage } & \multicolumn{5}{|c|}{ Storage } \\
\hline $\begin{array}{l}\text { Storage } \\
\text { System }\end{array}$ & Novo & 1 & $1.500,00$ & $1.500,00$ & $\begin{array}{l}\text { Storage } \\
\text { System }\end{array}$ & Novo & 1 & $1.500,00$ & $1.500,00$ \\
\hline \multicolumn{5}{|c|}{ Acessórios diversos } & \multicolumn{5}{|c|}{ Acessórios diversos } \\
\hline $\begin{array}{c}\text { Cabos, } \\
\text { Conectores, } \\
\text { etc. }\end{array}$ & Novo & 1 & 350,00 & 350,00 & $\begin{array}{c}\text { Cabos, } \\
\begin{array}{c}\text { Conectores, } \\
\text { etc. }\end{array} \\
\end{array}$ & Novo & 1 & 350,00 & 350,00 \\
\hline & TCOd1 & & & $83.150,00$ & & TCOd & & & $63.750,00$ \\
\hline
\end{tabular}


O valor total desta operação representaria um investimento no valor de $R \$$ 146.900,00 ( $T C O d=146900$ ), o que excederia o orçamento em $46,9 \%$, fato esse devido a uma falta de estudo prévio dos atuais equipamentos possíveis de serem utilizados, bem como de uma estrutura mal definida de compras na área de $\mathrm{Tl}$, o que geraria gastos excessivos além dos previstos. A fim de atenuar parcialmente o efeito desses fatores, o TCO1, Equação (2) pretende mostrar os efeitos de uma interferência, ainda que superficial para a compra dos equipamentos, em relação a estrutura orçamentária anterior,

$$
T \operatorname{co1}=\sum_{x=1}^{n} T \operatorname{Tco1x}\left\{\begin{array}{c}
\mathrm{TCO} 1>0 \\
T \cos x=\sum_{i=1}^{n} \sum_{e=1}^{n}(I T e)_{i}
\end{array}\right.
$$

para TCO11 relativo a SALA 1 e TCO12 a SALA 2. A Tabela 3 mostra que os resultados obtidos incluiram a reutilização de que diversos equipamentos já existentes na SALA 1, havendo apenas a reposição para suprir a demanda proposta para 0 número de estações de trabalho inicialmente planejada. 
Custo total de propriedade (TCO): Estudo de caso em uma instituição de ensino superior privada do Rio Grande do Sul

Alvaro Luiz Neuenfeldt Júnior, Charlene Coser Dalcol, Andreas Dittmar Weise, Julio Cezar Mairesse Siluk

Tabela 3 - TCO1 em relação a uma interferência superficial a atual estrutura

\begin{tabular}{|c|c|c|c|c|c|c|c|c|c|}
\hline \multicolumn{5}{|c|}{ SALA 1} & \multicolumn{5}{|c|}{ SALA 2} \\
\hline Descrição & $\begin{array}{c}\text { Statu } \\
\text { s }\end{array}$ & Qde & $\begin{array}{c}\text { Preço } \\
\text { Un. (R\$) }\end{array}$ & $\begin{array}{c}\text { Preço } \\
\text { Total (R\$) }\end{array}$ & Descrição & $\begin{array}{l}\text { Statu } \\
\mathbf{s}\end{array}$ & Qde & $\begin{array}{c}\text { Preço } \\
\text { Un. (R\$) }\end{array}$ & $\begin{array}{c}\text { Preço } \\
\text { Total (R\$) }\end{array}$ \\
\hline \multicolumn{5}{|c|}{ Desktop } & \multicolumn{5}{|c|}{ Desktop } \\
\hline $\begin{array}{l}\text { Processad } \\
\text { or Intel } \\
\text { Core } 2 \\
\text { Duo } 2.4 \\
\text { GHz; } 3 \\
\text { GB; HD } \\
160 \mathrm{~GB}\end{array}$ & $\begin{array}{l}\text { Usad } \\
0\end{array}$ & 20 & 600,00 & $12.000,00$ & $\begin{array}{c}\text { Processador } \\
\text { segunda } \\
\text { geração Intel } \\
\text { Core i3-2120 } \\
3.3 \mathrm{GHz}, 4 \\
\text { Threads; } 3 \\
\text { Mb; } 4 \text { GB; HD } \\
500 \mathrm{~GB}\end{array}$ & Novo & 30 & $\begin{array}{c}1.550,0 \\
0\end{array}$ & $46.500,00$ \\
\hline $\begin{array}{c}\text { Processad } \\
\text { or segunda } \\
\text { geração } \\
\text { Intel Core } \\
\text { i3-2120 } 3.3 \\
\text { GHz, } 4 \\
\text { Threads; } 3 \\
\text { Mb; } 4 \text { GB; } \\
\text { HD 500GB }\end{array}$ & Novo & 20 & $1.550,00$ & $31.000,00$ & - & - & - & - & - \\
\hline \multicolumn{5}{|c|}{ Monitor } & \multicolumn{5}{|c|}{ Monitor } \\
\hline LCD 17" & $\begin{array}{c}\text { Usad } \\
0\end{array}$ & 20 & 200,00 & $4.000,00$ & LCD 18,5" & Novo & 30 & 300,00 & $9.000,00$ \\
\hline LCD 18,5" & Novo & 20 & 300,00 & $6.000,00$ & - & - & - & - & - \\
\hline \multicolumn{5}{|c|}{ Servidor para aplicação de dados } & \multicolumn{5}{|c|}{ Servidor para aplicação de dados } \\
\hline $\begin{array}{l}\text { Processad } \\
\text { or Intel } \\
\text { Xeon } \\
\text { X3430 } 2.4 \\
\text { GHZ; } 6 \\
\text { GB; HD } \\
500 \text { GB }\end{array}$ & Novo & 1 & $2.900,00$ & $2.900,00$ & $\begin{array}{l}\text { Processador } \\
\text { Intel Xeon } \\
\text { X3430 } 2.4 \\
\text { GHZ; } 6 \text { GB; } \\
\text { HD } 500 \text { GB }\end{array}$ & Novo & 1 & $\begin{array}{c}2.900,0 \\
0\end{array}$ & $2.900,00$ \\
\hline \multicolumn{5}{|c|}{ Switch } & \multicolumn{5}{|c|}{ Switch } \\
\hline $\begin{array}{c}24 \text { portas } \\
10 / 100 \\
\text { Mbps; } 3.2 \\
\text { GHz de } \\
\text { banda }\end{array}$ & Novo & 2 & 250,00 & 500,00 & $\begin{array}{c}24 \text { portas } \\
10 / 100 \mathrm{Mbps} ; \\
3.2 \mathrm{GHz} \text { de } \\
\text { banda }\end{array}$ & Novo & 2 & 250,00 & 500,00 \\
\hline \multicolumn{5}{|c|}{ Nobreak } & \multicolumn{5}{|c|}{ Nobreak } \\
\hline $\begin{array}{c}1300 \mathrm{Va} \\
\text { com duas } \\
\text { baterias } \\
1300 \mathrm{Va}\end{array}$ & $\begin{array}{l}\text { Usad } \\
0\end{array}$ & 4 & 500,00 & $2.000,00$ & $\begin{array}{l}1300 \text { Va com } \\
\text { duas baterias }\end{array}$ & Novo & 6 & 500,00 & $3.000,00$ \\
\hline $\begin{array}{c}\text { com duas } \\
\text { baterias }\end{array}$ & Novo & 4 & 500,00 & $2.000,00$ & - & - & - & - & - \\
\hline \multicolumn{5}{|c|}{ Storage } & \multicolumn{5}{|c|}{ Storage } \\
\hline
\end{tabular}


Custo total de propriedade (TCO): Estudo de caso em uma instituição de ensino superior privada do Rio Grande do Sul

Alvaro Luiz Neuenfeldt Júnior, Charlene Coser Dalcol, Andreas Dittmar Weise, Julio Cezar Mairesse Siluk

\begin{tabular}{|c|c|c|c|c|c|c|c|c|c|}
\hline $\begin{array}{l}\text { Storage } \\
\text { System }\end{array}$ & $\begin{array}{c}\text { Usad } \\
0\end{array}$ & 1 & 800,00 & 800,00 & $\begin{array}{l}\text { Storage } \\
\text { System }\end{array}$ & Novo & 1 & $\begin{array}{c}1.500,0 \\
0\end{array}$ & $1.500,00$ \\
\hline \multicolumn{5}{|c|}{ Acessórios diversos } & \multicolumn{5}{|c|}{ Acessórios diversos } \\
\hline $\begin{array}{l}\text { Cabos, } \\
\text { Conectore } \\
\text { s, etc. }\end{array}$ & Novo & 1 & 350,00 & 350,00 & $\begin{array}{c}\text { Cabos, } \\
\text { Conectores, } \\
\text { etc. }\end{array}$ & Novo & 1 & 350,00 & 350,00 \\
\hline \multicolumn{4}{|c|}{ TC011 } & $42.750,00$ & \multicolumn{4}{|c|}{ TC012 } & $63.750,00$ \\
\hline \multicolumn{4}{|c|}{ Total Reutilizado } & $18.800,00$ & \multicolumn{4}{|c|}{ Total Reutilizado } & - \\
\hline
\end{tabular}

Fonte: Dados da pesquisa.

Para a SALA 2, de acordo com o explicitado anteriormente, não existem quaisquer tipos de equipamentos, logo devem ser comprados novos, o que resulta em um dispêndio financeiro superior em $49 \%$ ao existente para a SALA 1. Dessa forma, a soma dos valores para ambos laboratórios resultou em um total de investimentos necessários equivalente a $\mathrm{R} \$ 106.500,00 \quad$ (TCO1=106500), 6,5\% superior ao inicialmente proposto $($ TCO $i=100000)$.

Como o TCO1 ainda não atingiu a meta, foi idealizada uma segunda alternativa de investimentos, denominada $\mathrm{TCO} 2$, que contempla, além da reutilização dos equipametos já existentes na SALA 1, a substituição da compra de novos Desktops por processadores chamados de Thin Clients, de acordo com a Equação (3),

$$
\operatorname{Tco} 2=\sum_{x=1}^{n} T \operatorname{co2} x\left\{\begin{array}{c}
\mathrm{TCO2}>0 \\
\text { TCO2x }=\sum_{i=1}^{n} \sum_{e=1}^{n}(I T e)_{i}
\end{array}\right.
$$

para TCO21 referente a SALA 1 e TCO22 a SALA 2, cujos resultados estão expostos na Tabela 4. 
Custo total de propriedade (TCO): Estudo de caso em uma instituição de ensino superior privada do Rio Grande do Sul

Alvaro Luiz Neuenfeldt Júnior, Charlene Coser Dalcol, Andreas Dittmar Weise, Julio Cezar Mairesse Siluk

Tabela 4 - TCO2 relativo a alternativas para o TCO1

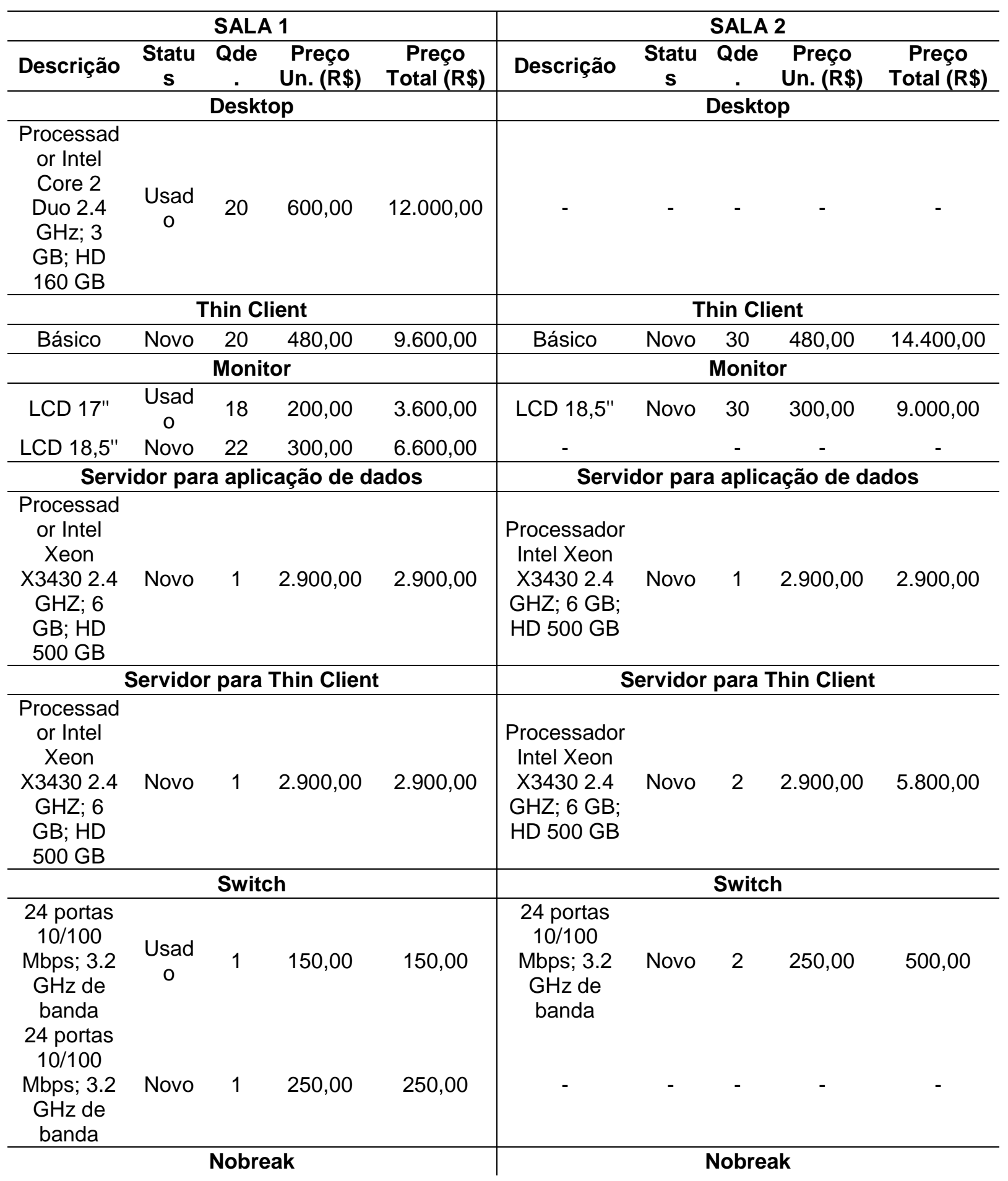


Custo total de propriedade (TCO): Estudo de caso em uma instituição de ensino superior privada do Rio Grande do Sul

Alvaro Luiz Neuenfeldt Júnior, Charlene Coser Dalcol, Andreas Dittmar Weise, Julio Cezar Mairesse Siluk

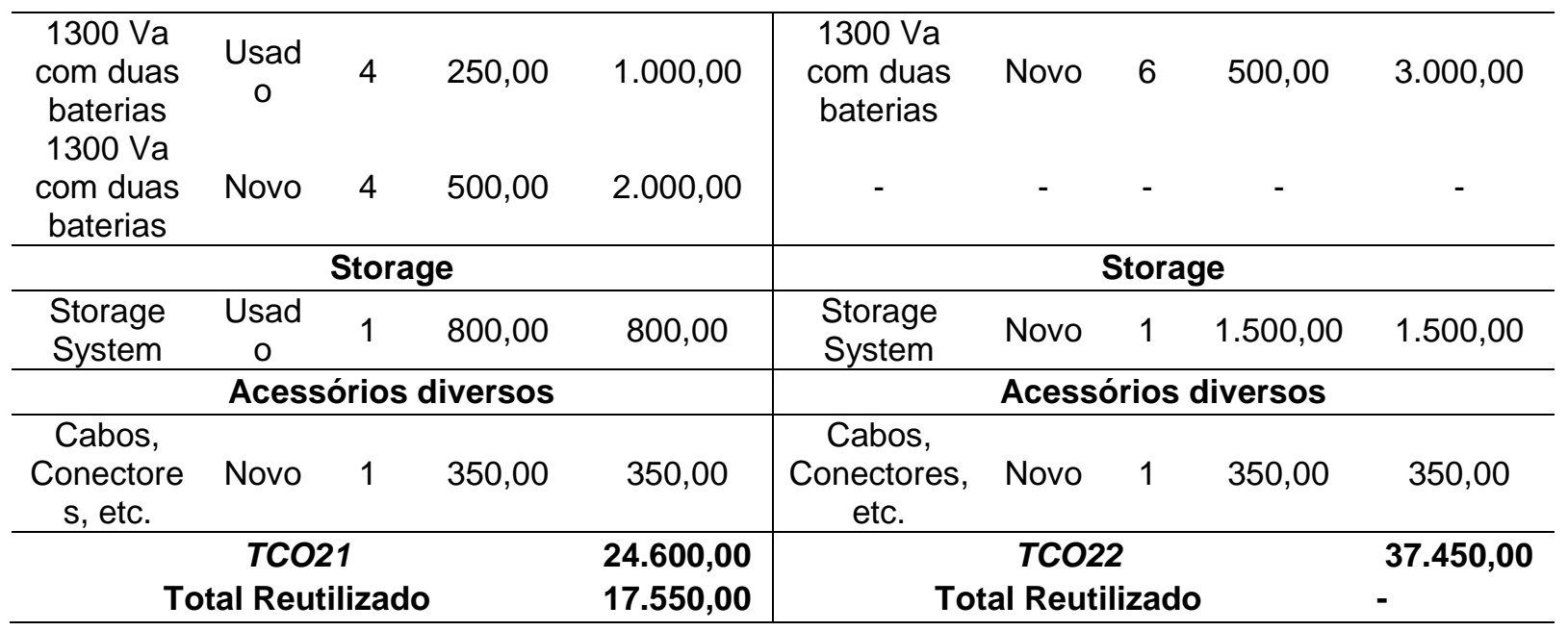

Fonte: Dados da pesquisa.

Nota-se que a proposta contempla a utilização de aparelhos denominados por Thin Clients (computador cliente através da sua relação direta com um servidor, em cuja estrutura não é instalado praticamente nenhum aplicativo para sua operação), os quais tecnicamente estão de acordo com as necessidades de utilização requisitadas, apesar do seu nível reduzido de processamento em relação aos tradicionais Desktops.

Com base nessa substituição tem-se uma redução no custo total de obtenção dos equipamentos para $R \$ 62.050,00$ (TCO2=62050), o que em termos gerais significa uma redução em $38 \%$ do valor para investimentos proposto inicialmente, mesmo havendo a necessidade da compra de um servidor extra para os Thin Clients. A fim de facilitar a compreensão dos valores obtidos, apresenta-se no Gráfico 1 os TCOs calculados em relação ao proposto. 


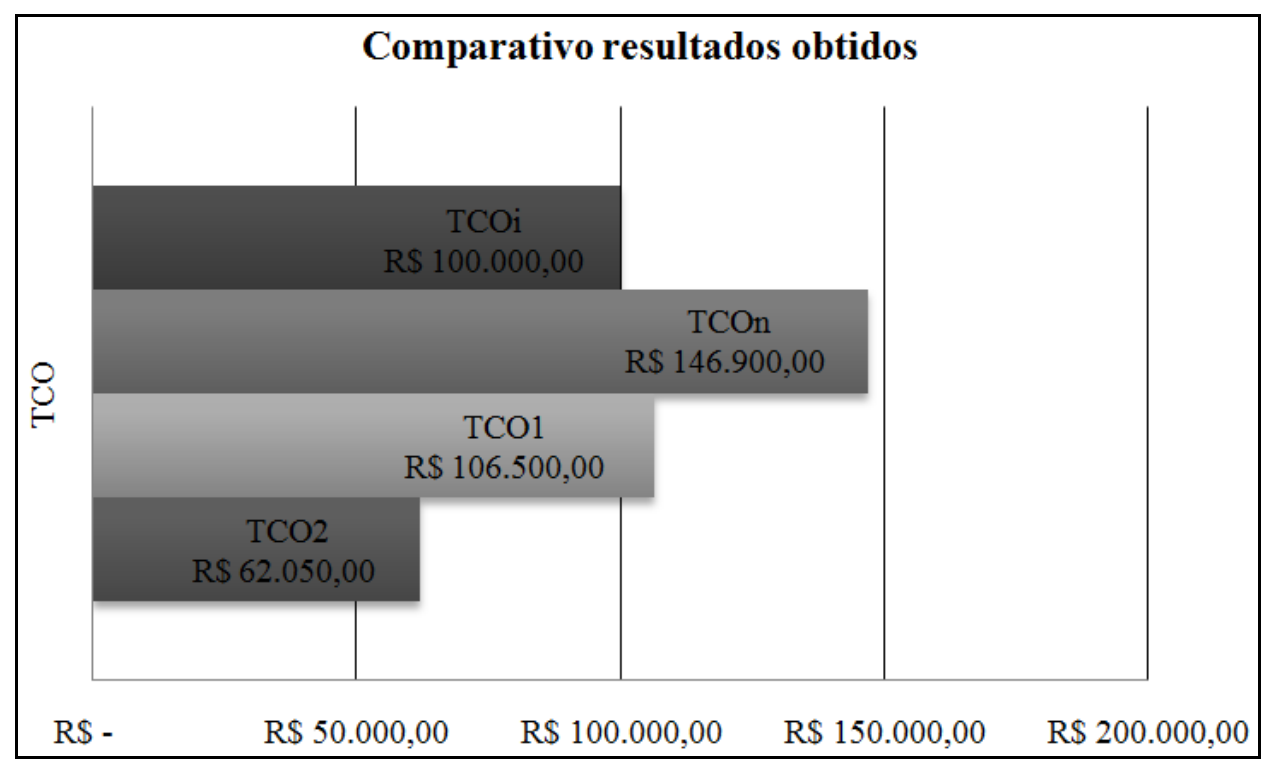

Gráfico 1 - Resultados obtidos com o estudo

Fonte: Dados da pesquisa.

Através desse gráfico percebe-se a mudança ocorrida dos valores onde, desde a TCOd até o TCO2 houve uma otimização do valor a ser investido equivalente a $58 \%$ do montante inicialmente considerado, gerando uma possível economia ao final das compras de equipamentos igual a $R \$ 84.850,00$, adequando-se, portanto, ao valor a ser investido ao TCOi considerado pela direção da IES, atendendo satisfatoriamente os requisitos estabelecidos para a construção de ambos os laboratórios de informática.

Em relação ao TCO processos, conforme descrito anteriormente, não havia um fluxo de compra dos materiais para as solicitações da área de TI padronizada, sendo proposto inicialmente o estudo da maneira como esta era habitualmente abordada pelos colaboradores da instituição, segundo mostra a Figura 1. 
Alvaro Luiz Neuenfeldt Júnior, Charlene Coser Dalcol, Andreas Dittmar Weise, Julio Cezar Mairesse Siluk

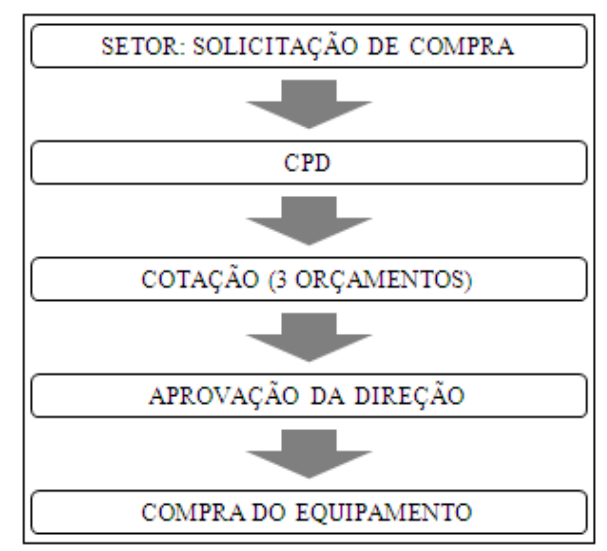

Figura 1 - Processos de solicitação para TI atuais Fonte: Elaborado pelos autores.

Nesse fluxo, a compra de equipamentos iniciava com a solicitação por parte do interessado ao Centro de Processamentos de Dados (CPD) da IES. Este por sua vez realizava três cotações simples de orçamentos com empresas escolhidas de maneira aleatória, obtendo por fim um documento submetido para a aprovação do corpo diretivo da instituição, havendo a solicitação deste para o fornecedor.

Em nenhum momento constam elementos que tratam o relacionamento com 0 fornecedor, tão pouco do controle das últimas compras realizadas pela instituição, não havendo um controle dos equipamentos adquiridos, do seu uso ou do nível de obsolescência atual. Além dos pontos observados, atualmente a prestação de manutenção dos equipamentos ocorre de maneira a atender a demanda, seguindo um procedimento de contratação informal, onde cada setor da instituição aplica seus recursos próprios para a obtenção do serviço, não havendo um controle centralizado dessas informações.

Visando a melhoria dos procedimentos atuais, para o TCO processos foi proposto um novo procedimento para a compra e solicitação de serviços em TI, conforme mostra a Figura 2. 
Alvaro Luiz Neuenfeldt Júnior, Charlene Coser Dalcol, Andreas Dittmar Weise, Julio Cezar Mairesse Siluk

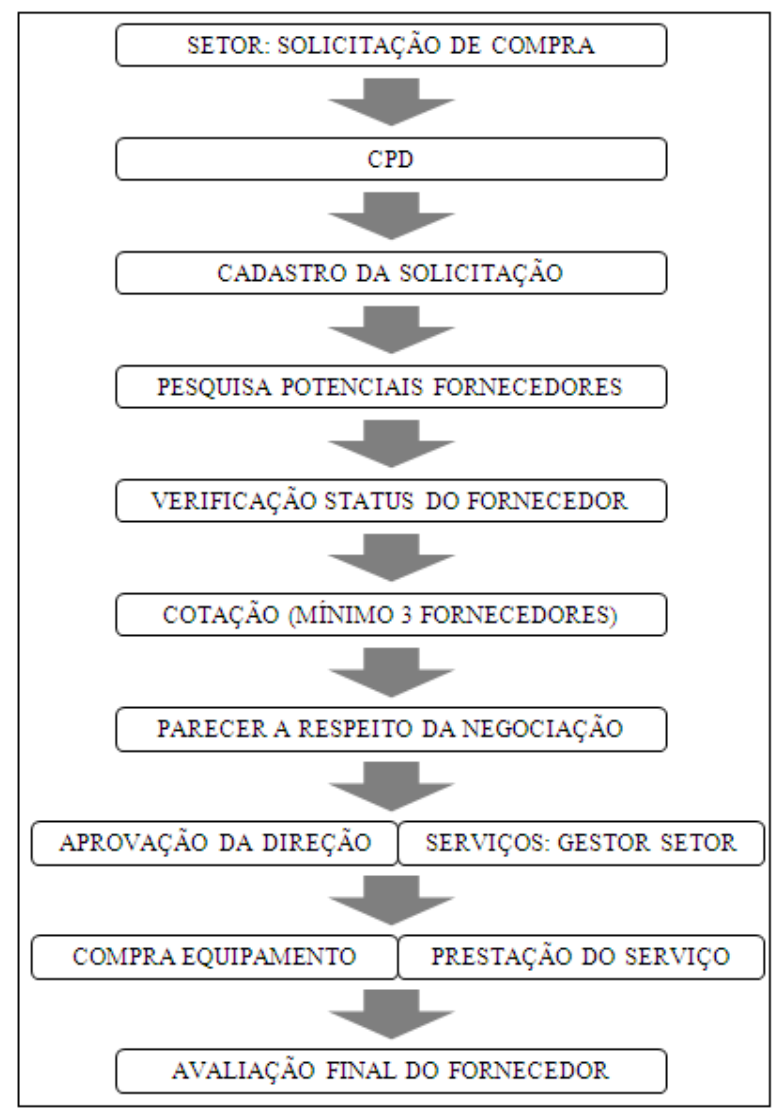

Figura 2 - TCO para a mudança dos processos de pedidos de TI Fonte: Elaborado pelos autores.

A maior diferença entre o processo atual e o proposto é na forma com que ocorre a tratativa entre o CPD e o(s) fornecedor(es), onde ao invés de haver uma relação unicamente de cotação dos preços, há uma preocupação com a maneira como esta será monitorada, a fim de em futuras negociações se conhecer o patamar no qual ele se encontra em relação à IES. Além disso, incorporou-se ao fluxo de solicitação de produto o de prestação de serviços, havendo apenas uma diferença nos responsáveis pela aprovação, pois enquanto o primeiro exigia um maior cuidado por questões financeiras, o segundo está mais relacionado à rotina dos processos do CPD da instituição, sendo o valor retirado diretamente do centro de custo do setor solicitante, não havendo a necessidade do acionamento direto dos diretores da empresa. 
Alvaro Luiz Neuenfeldt Júnior, Charlene Coser Dalcol, Andreas Dittmar Weise, Julio Cezar Mairesse Siluk

Outro ponto a se ressaltar é que as cotações não necessariamente devem estar vinculadas a, no máximo, três fornecedores como no caso atual, abrindo a possibilidade de haver diversas negociações com estes, abrindo precedentes para a obtenção de um melhor custo-benefício durante a solicitação do equipamento ou serviço. O cadastro, bem como a gestão dessas informações, pode estar vinculado até mesmo em uma planilha eletrônica simples, pois a demanda por esse tipo de serviço, por parte da IES, não é elevado.

\section{CONCLUSÃO}

O estudo teve como objetivo mostrar a aplicação do Custo Total de Propriedade na reestruturação do setor de compras de $\mathrm{Tl}$ em uma Instituição de Ensino Superior privada localizada no Rio Grande do Sul, partindo inicialmente do caso da expansão de um de seus laboratórios de informática e da criação de uma nova sala para a modalidade de Ensino à Distância (EAD). Nesse sentido, acredita-se ter atingido satisfatoriamente as expectativas, oferecendo aos interessados uma visão mais sistêmica dos possíveis caminhos ótimos a serem seguidos nos próximos processos de compra da IES. Outro ponto a se destacar se refere à facilidade de aplicação do TCO para esse fim, tornando o trabalho de quem organiza esse tipo de projeto mais simples, padronizado e dinâmico, retornando respostas rápidas sobre o comportamento dos investimentos.

Quanto às duas propostas de TCO desenvolvidas, a de otimização dos custos e a de processos, notou-se em relação à primeira uma redução no valor de $\mathrm{R} \$$ $100.000,00$ proposto pela direção da instituição de aproximadamente $38 \%$, gerado principalmente devido ao estudo de maneiras alternativas de se atender os usuários, como o caso dos Thin Clients, que oferecem um serviço com uma qualidade similar aos tradicionais Desktops, porém com uma economia por equipamento de $70 \%$, o que se refletiu diretamente no valor final total obtido para o TCO2 (TCO2=62.050,00). 
Alvaro Luiz Neuenfeldt Júnior, Charlene Coser Dalcol, Andreas Dittmar Weise, Julio Cezar Mairesse Siluk

Com relação à segunda questão, relacionada ao estudo dos processos de compra ou solicitação de serviços para TI, pode-se futuramente propor uma nova metodologia que contemple um estudo mais amplo sobre os dados qualitativos de cada fornecedor antes da solicitação, bem como da elaboração, após a aquisição, de relatórios contendo o nível obtido de custo-benefício na negociação, gerando assim um amplo histórico a respeito de cada um dos fornecedores, guiando a instituição em futuras solicitações.

Em relação à utilização do TCO, cabe a discussão acerca do papel do envolvimento das pessoas ao contexto de mudanças abordado, de maneira que uma possível falta de apoio dos mesmos nesse processo certamente resultará em distorções na proposta originalmente planejada pela gestão da empresa. Outro ponto relevante e que também pode se tornar um potencial limitante na sua aplicação, é a falta de metodologias pré-estabelecidas capazes de servir como guia didático de implementação, visto a sua natureza de concepção genérica a qualquer formato de empreendimento, além de aplicações que envolvam um âmbito mais científico de verificação dos problemas, o que pode tornar a sua utilização desconexa com a realidade.

Para trabalhos futuros, sugere-se a aplicação do TCO não somente para os projetos que envolvam a compra de equipamentos de $\mathrm{Tl}$, abrangendo assim outras formas de aquisição de materiais, conforme as características da IES ou outra entidade estudada.

\section{REFERÊNCIAS}

ALMEIDA, K.D.M.; BORGES, M.F.; CARVALHO, C.V. A \& LIMA JUNIOR, J.A.T. (2010). Custo Total de Propriedade aplicado ao Centro de Documentação Histórica da Universidade Severino Sombra: Um estudo de caso. Revista TECCEN, 3 (1):1-21.

BOK, D. (2003). Universities in the marketplace: the commercialization of higher education. Princeton, Princeton University. 
Alvaro Luiz Neuenfeldt Júnior, Charlene Coser Dalcol, Andreas Dittmar Weise, Julio Cezar Mairesse Siluk

COUSINS, P.D.; LAMMING, R.C.; LAWSON, B. \& SQUIRE, B. (2008). Strategic Supply Management. Harlow, Pearson Education.

DEGRAEVE, Z.; ROODHOOFT, F. E. \& VAN DOVEREN, B. (2005). The use of total cost of ownership for strategic procurement: a company-wide management information system. Journal of the Operational Research Society. 56:51-59.

DIAS SOBRINHO, J.A. (2003). Universidade na encruzilhada. In: Seminário Universidade: por que e como reformar? Brasília: UNESCO Brasil, Ministério da Educação.

ELLRAM, L.M. (1993). Total cost of ownership: Elements and implementation. International Journal of Purchasing and Materials Management. 29:3-11.

ELLRAM, L.M\& ZSIDISIN, G. (2002). Factor that drive purchasing and Supply Management's Use of Information Technology. IEE Transaction on Engineering Management. 49(3):269-281.

FEIMAN, J. (2012). Application TCO Model: Categories and Architectures. Disponível em: http://www.gartner.com Acesso em 15/set/2012.

FERRIN, B.G. \&PLANK, R.E. (2002). Total cost of ownership models: An exploratory study. Journal of Supply Chain Management. 38(3):18-29.

GARFAMY, R.M. (2006). A data envelopment analysis approach based on total cost of ownership for supplier selection. Journal of Enterprise Information Management. 19(6): 662-678.

GIL. A.C. (2010). Como elaborar projetos de pesquisa. São Paulo: Atlas.

HANSEN, G.L. (2001). Limites e Potenciais das Novas Modalidades de Gestão Universitária. In: ALMEIDA, M. (Org.). A Universidade Possível: experiências da gestão universitária. São Paulo: Editores Associados.

HURKENS, C., VALK, W. \&WYNSTRA, F. (2006). Total cost of ownership in the services industry: a case study. Journal of Supply Chain Management. 42(1):27-37.

INEP - INSTITUTO NACIONAL DE ESTUDOS E PESQUISAS EDUCACIONAIS ANÍSIO TEIXEIRA. Sinopse da Educação Superior. Disponível em: <http://www.inep.gov.br/superior/censosuperior/sinopse/>. Acesso em: 15/set/2012. 
Alvaro Luiz Neuenfeldt Júnior, Charlene Coser Dalcol, Andreas Dittmar Weise, Julio Cezar Mairesse Siluk

KOSIER, J.M. \& STRONG, D. (2006). Supply/demand chain modeling utilizing logisticalbased costing. Journal of Enterprise Information Management. 19(3):346-360.

KRAEMER, M.E.P. A Universidade do século XXI rumo ao desenvolvimento sustentável. Disponível: <http://www.gestaoambiental.com.br.php>. Acesso em 06/set/2012.

KULMUALA, H.I. (2004). Developing cost management in customer-supplier relationships: three case studies. Journal of Purchasing and Supply Management. 10:65-77.

LAKATOS, E.M. \& MARCONI, M.A. (2001). Metodologia do trabalho científico: procedimentos básicos, pesquisa bibliográfica, projeto e relatório publicações e trabalhos científicos. São Paulo: Atlas.

LIMA FILHO, R.N.; BRUNI, A.L.; SAMPAIO, M.S., PEREIRA, A.G. (2011). Conceitos relevantes de Custos: A visão de textos didáticos, o olhar da Teoria da Contabilidade e a percepção de discentes. ABCustos, Associação Brasileira de Custos .6(3).

MCCHLERY, S. \& ROLF, T. (2004). University Costing Systems: a case study on value management. Journal of Finance and Management in Public Sciences. 4(1).

MENEGAT, V. (2006). Utilização dos sistemas de custos pelas Instituições de Ensino Superior do Sistema ACAFE - SC. In: 6 Congresso USP de Controladoria e Contabilidade. São Paulo, USP, 2006.

MERLO, R.A. \& BEUREN, I.M. (2004). Sistema de informações para a gestão econômico-financeira de uma universidade comunitária: um estudo de caso da UNOESC. In: BEUREN, Ilse Maria (Org.) Sistema de informações para a gestão universitária. São Miguel do Oeste, UNOESC, 2004.

MINAYO, M.C.S. (2010). Pesquisa Social: teoria, método e criatividade. Petrópolis, Vozes.

OGDEN, J.A.; ZSIDISIN, G.A. \& HENDRICK, T.E. (2002). Factors that influence chief purchasing officer compensation. The Journal of Supply Chain Management. 38(3):3038.

PARK, J.; SHIN, K.; CHANG, T.W. \& PARK, J. (2010). An integrative framework for supplier relationship management. Industrial Management \& Data Systems. 110(4):495515. 
Alvaro Luiz Neuenfeldt Júnior, Charlene Coser Dalcol, Andreas Dittmar Weise, Julio Cezar Mairesse Siluk

RAMOS, M.M. (2004). Interaction between management accounting and supply chain management. Supply Chain Management: An international Journal. 9(2):134-138.

SANTOS, S.M.S; BORNIA, A.C.; LEITE, M.S.A. (2010). Sistema de Gestão de Custos Associado à Cadeia de Valor. ABCustos, Associação Brasileira de Custos. 5(1).

TOMKINS, C. (2001) .Interdependencies trust and information in relationships alliances and networks. Accounting Organizations and Society. 26(2):161-191.

WOUTERS, M.; ANDERSON, J.C. \& WYSNSTRA, F. (2005). The adoption of total cost of ownership for sourcing decisions: a structural equations analysis. Accounting Organizations and Society. 30(2):167-91.

YIN, R.K. (2009). Case Study Research: Design and Methods. Sage: Thousand Oaks.

ZACHARIASSEN F. \& ARLBJØRN J. S. (2011). Exploring a differentiated approach to total cost of ownership. Industrial Management \& Data Systems. 111(3):448-469.

Data de Submissão: 21/11/2012

Data de Aceite: 18/05/2014 